\section{The role of theragnostics in oncology: an interview with Stefano Buono}

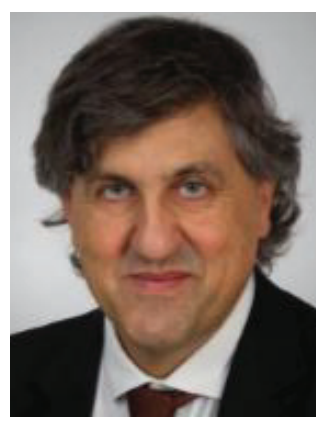

Stefano Buono* speaks to Commissioning Editor, Sebastian Dennis-Beron: Stefano Buono is the Chief Executive Officer, a member of the board of directors, and a founder of Advanced Accelerator Applications (AAA). He has been responsible for overseeing AAA operations, including the development and commercialization of the current portfolio of diagnostic and therapeutic molecular nuclear medicine products, and listing AAA on Nasdaq (stock symbol: AAAP). Prior to founding the company in 2002, he worked as a physicist at the Centre for Advanced Studies, Research and Development, or CRS4, in Italy. During his 6-year tenure with CRS4, he headed a team of engineers working on different international research projects in the field of energy production and nuclear waste transmutation. Before working at CRS4, and alongside his appointment at CRS4, he worked with Physics Nobel Laureate Carlo Rubbia at The European Organization for Nuclear Research (CERN), the world's largest research laboratory for particle physics, in Geneva, Switzerland. He actively participated in the development of CERN's Adiabatic Resonance Crossing (ARC) method. He is the author of numerous scientific papers. He received his Master's degree in Physics from the University of Turin, in Italy, in 1991.

Accepted for publication: 28 April 2017; Published online: 9 June 2017

Q Could you please provide a brief summary of your career to date? What triggered your interest in nuclear medicine?

Before founding Advanced Accelerator Applications (AAA) in 2002, I was a researcher in the nuclear energy field, and together with Carlo Rubbia and a team at the European Organization for Nuclear Research (CERN) in Switzerland, developed a patent that had an application in nuclear medicine. As our research began to slow down, I wanted to understand whether there was a possibility to develop the patent, and started to look at the field of nuclear medicine. Through my investigation into the field, I realized there was a need for the drug that was an output of our research - a drug for Positron Emission Tomography (PET), called fludeoxyglucose, or FDG. Fludeoxyglucose was developed with the use of particle accelerators, machines that I was familiar with, given my previous career. So, I became interested in that project and ultimately decided to start a company to develop this drug, as well as carry on the research that was coming out of the patents from CERN.

\section{Q What was your vision for AAA at its inception?}

My original vision, and one we are still implementing today, was to develop these patents into new therapeutic applications that can benefit from advances in nuclear

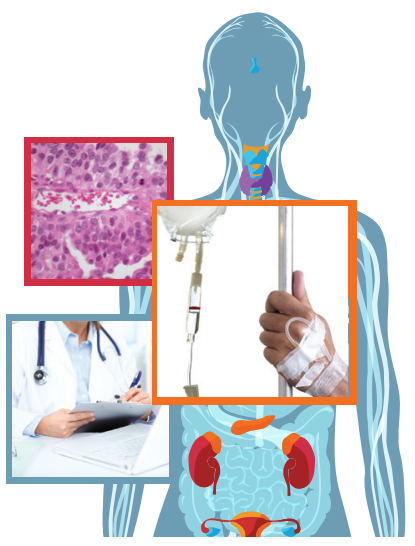

International Journal of Endocrine Oncology

\section{KEYWORDS}

- NETs • nuclear medicine

- theragnostics

'Advanced Accelerator Applications, 350 Fifth Avenue, Suite 6902, New York, NY, 10118, USA

*Author for correspondence: rachel.levine@adacap.com 
medicine. The first drugs we developed, however, were diagnostics, in keeping with the rest of the field. Before entering into therapeutics, we first needed to master the manufacturing, development and logistical challenges of an industry where products have shelf lives of hours or days.

This strategy was aligned with my idea to fund the company not only with money from our shareholders, but also with revenues from these diagnostic drugs, so we could support development efforts required to enter the field of therapeutics. This has been the goal from the beginning and it is still very much the case for the company today.

We developed lutetium Lu 177 dotatate $\left(\right.$ Lutathera $\left.^{\circledR}\right)$, our lead investigational therapeutic, because of our interest in getting involved with therapy, and the fact that we found a very interesting candidate in 2008. We acquired the intellectual property through the acquisition of a company in 2010, and we started to really push therapeutics onto the main stage starting that year. Achieving marketing authorization for this drug has been one of our focuses for approximately the past 15 years.

\section{Q Theragnostics is a key patient} management strategy in precision medicine today. How have theragnostics evolved in your time at AAA?

Over the years, our vision has developed into the use of diagnostics and therapy together (theragnostics), for better management of cancer patients. We saw the possibility of using exactly the same molecular targeting compound to create both diagnostic and therapeutic drugs. This is the case with lutetium Lu 177 dotatate (Lutathera) and its complementary diagnostic, which has already been approved in the US as NETSPOT ${ }^{\circledR}$, and in Europe as SomaKit TOCTM.

This concept was key to our vision - using this first pairing of theragnostic drugs as an example, we are able to really change the management of patients. One study we ran with the diagnostic product showed an ability to change patient management decisions in $71 \%$ of the cases, an outstanding number. This demonstrates the importance of measuring all of the patient's statistics, to better understand their disease. In addition, it allows us to select patients for the therapy, and design a treatment plan and follow-up protocol.

This is a very unique approach. Only nuclear medicine gives you the possibility of using exactly the same molecular targeting compound for both diagnostic and therapeutic purposes. The only difference between the two is the type of radioisotope attached to the targeting molecule. When we talk about theragnostics in technical terms, this is just the coupling of in vivo diagnostics with therapy, versus an in vitro lab diagnostic approach used in other fields. Our pipeline now includes several theragnostic drug pairings, because we saw a lot of opportunities in the field, and at the moment, we are the only operational company in nuclear medicine that is investing in development of these pairings.

Another drug candidate we added to our theragnostic pipeline is a urea-based ligand to prostatespecific membrane antigen (PSMA) that is used in the management of prostate cancer. Despite the fact that we have a compound that will be moving into Phase I clinical trials, this is actually a much more mature project, because we have seen a lot of very similar compounds already being used in diagnosing and treating prostate cancer patients. Several examples of this can be found in academia in Germany, where three similar compounds have been produced. This provides a lot of guidance in terms of our development, although they are not using the same targeting compound for both diagnosis and therapy, which is our strategy. We are developing a PSMA compound that we inlicensed from Johns Hopkins University (MD, USA), which behaves in similar ways.

The third drug pairing in our theragnostic pipeline is based on a bombesin antagonist, which has demonstrated potential to address gastrointestinal stromal tumors (GIST), breast cancer and prostate cancer by targeting a different molecular receptor.

In general, we are searching for targets that are specific to certain tumors, to which we can attach our platform of isotopes for either diagnosis or treatment. This includes lutetium 177, which emits electrons that give it the capacity to kill tumors a few millimeters away, once the drug has reached the lesion; and gallium 68, the second isotope in the platform, which is used for PET. Gallium 68 is the isotope used in NETSPOT/SomaKit TOC, our approved diagnostic drugs used for visualizing neuroendocrine tumors (NETs).

\section{Q Can you give us an idea of the mechanics of peptide receptor radionuclide therapies in cancers, \& particularly the mechanism of Lutathera in neuroendocrine tumors?}

In general terms, you have a targeting vector carrying a radioactive payload, which is trapped 
inside the targeting molecule, so it is not seen in the extracellular space and therefore not interacting with the body. That is why the diagnostics and therapeutics behave exactly the same way inside the body. Their different modes of action are determined by the nature of the radioisotopes attached to them (i.e. gallium 68 for diagnostics or lutetium 177 for therapy), but this does not come into play until they have reached the target lesion(s).

Once the molecular target receptor is reached, the drug is internalized by the tumor cell. In the case of the therapeutic, it must stay inside the tumor cell for a few days, so the radiation is released, ultimately killing the tumor cell.

In the case of lutetium Lu 177 dotatate (Lutathera $^{\circledR}$ ) for NETs, the targeting vector used is a peptide analogue of somatostatin, since somatostatin receptors are highly expressed in most NETs. The drug reaches the NET due to its very high affinity to these somatostatin receptors, where it is then internalized. The targeting molecule is cleared from the rest of the body very fast (in a matter of a few hours), but the radioisotope is treating the tumor from the inside for many days.

Interestingly, the lutetium 177 isotope used for therapy also emits some gamma rays, which are used for imaging purposes. Meaning, if you take PET images of a patient immediately after treatment, or even up to 7 days after, you are still able to see radiation from the drug inside the tumor(s), even though the rest of the body is clear. This specificity is why our investigational therapy, lutetium Lu 177 dotatate (Lutathera), has demonstrated such impressive results in its Phase III trial.

\section{Q Lutathera was recently evaluated in the} NETTER-1 Phase III trial. Can you give our readers a brief outline of the trial?

We designed the trial in inoperable, progressive, somatostatin receptor positive midgut NETs against Octreotide LAR as the comparator. Octreotide LAR was chosen because at the time of the trial, that was the only approved drug which showed efficacy in controlling the tumors being studied.

We wanted a population that really progressed while under the standard of care, which at the time was somatostatin analogs. The regulatory authorities asked us to use double the dose of Octreotide LAR as a comparator, so we did not have a placebo arm.
Once we ran the study, the median progressionfree survival (PFS) for the comparator was achieved after 8.6 months, while median PFS for lutetium Lu 177 dotatate (Lutathera) was not reached during the initial analysis. In the final analysis, we achieved a hazard ratio of 0.21 , which is very significant for an oncology drug, with PFS in the region of approximately $30-40$ months. This is a $79 \%$ reduction in the risk of disease progression or death. A preplanned interim overall survival analysis determined that compared to the Octreotide LAR arm, our treatment led to a $60 \%$ reduction in the estimated risk of death with a very strong hazard ratio of 0.40 and a low $p$-value of $p=0.004$. This was a very good sign of efficacy for overall survival. That is the reason why the study received strong media coverage when the results were presented at ESMO in 2015. We received the same level of enthusiasm and coverage for almost a full year at all of the main oncology conferences.

More recently, we presented quality of life data, which may be of real interest to the community, because the data suggest that the drug also improved several quality of life factors. We measured many quality of life variables, with almost all of them showing some improvement, and achieved statistically significant improvement in key areas like diarrhea, which is a severe symptom of midgut NETs, and global health status.

\section{Q What do the results mean concretely for GEP-NET patients \& physicians?}

Well, we believe this means that there is now an investigational drug that distinguishes itself in terms of efficacy. In basic terms, there are currently a couple of approved drugs available that result in PFS improvements of approximately 11 months, compared to our investigational drug that offers an improvement of approximately 30-40 months. This is a potentially huge development that could completely change disease management for these patients. Going one step further, if we can add lutetium Lu 177 dotatate (Lutathera) as an option for second line treatment in these patients, alongside NETSPOT/ SomaKit TOC, the complementary diagnostic test, this may contribute greatly to improved outcomes for this disease.

\section{Following the NETTER-1 trial, what are the next steps for the therapeutic?}

We are very close to re-submission of our New Drug Application (NDA) for lutetium Lu 177 
dotatate (Lutathera) to the US FDA. We had some issues with the formatting of the clinical datasets that we had previously provided to the FDA, and the reviewers were not able to reproduce the data file, which prevented them from performing their required independent analysis. So, we had to revise the datasets and are aiming to resubmit our NDA by mid-2017. As a result, we could potentially receive marketing authorization in the US around the end of the year.

The European Medicines Agency does not need to independently repeat the data analysis, so we may potentially receive marketing authorization in Europe in the third quarter of this year. We hope that by the end of 2017 , or the beginning of 2018, the drug can be formally made available to the patient community.

There is already some degree of availability for the drug; as we have compassionate use and named patient programs in 11 countries, through which more than 1,700 patients have been treated already. Most of these patients have been treated in Europe, because we only initiated an Expanded Access Program in the US, in July of 2016. We already have more than 100 patients that have been included into the US program so far. So, even if the drug is not yet commercially available, we are making it available in 17 centers in the US and more than 70 centers in Europe, so patients with an urgent need for the drug have access to it.

What are some of the additional products under development in AAA's expanding theragnostics pipeline?

The theragnostics concept is very important to us, as we are already seeing strides in the potential use of theragnostics in the field of prostate cancer. Much like in the case of lutetium Lu 177 dotatate (Lutathera) and NETSPOT/SomaKit TOC, we have several examples in Germany by a community of doctors, who themselves have developed a new compound using a urea-based PSMA-ligand. This ligand is becoming a diagnostic standard for prostate cancer in Germany, but it is also a drug that has already been used to provide hundreds of patients with palliative and terminal care, exhibiting very strong activity. As a result, we decided to license a very similar compound from John Hopkins University into our pipeline, and we will be moving into clinical trials this year for both the diagnostic and the therapy.
Another compound we have in our pipeline is a bombesin antagonist, which targets gastrin releasing peptide receptors (GRPR), which we are developing for GIST, breast cancer, and prostate cancer. There is an ongoing study with the diagnostic version of this drug in GIST patients, and our goal is to have a therapeutic trial started by the end of the year.

Where do you see theragnostics and the use of peptide receptor radionuclide therapies \& molecular nuclear medicine in oncology going in the future?

I think that nuclear medicine, and particularly theragnostics, will become a key part of patient management in the future, because of their many advantages. I believe we have shown with NETSPOT/SomaKit TOC and lutetium Lu 177 dotatate (Lutathera) that this approach may add a lot to NET patient management, from initial diagnosis, through to the therapeutic protocol and follow-up. We see the same potential benefits in prostate cancer treated with the PSMA-ligand, as the mechanism of action is very similar.

We need to be very good in how we target cancer, but we do not need a new mechanism of action for these drugs to be effective, because the mechanism of the radioisotopes is always the same: gallium 68 radiation results in the imaging used for diagnosis and monitoring, and the lutetium 177 radiation kills the tumor. We have the potential of using this same concept for many other cancers, provided we are able to develop good molecular targeting molecules with sufficient clearance of these compounds from the body after their use. We are constantly trying to find more novel targets, so we see a very bright future for theragnostics.

\section{Closing statement}

Nuclear medicine has been around for many years, much like immuno-oncology, but I think there are certain triggers that give all disciplines greater importance at any given moment. The recent discovery of the effects of checkpoint inhibitors has been very important for immuno-oncology, and I sense that the theragnostic approach represented by lutetium Lu 177 dotatate (Lutathera) and NETSPOT/SomaKit TOC will trigger a similar new era for nuclear medicine.

\section{Disclaimer}

The opinions expressed in this interview are those of the interviewee and do not necessarily reflect the views of Future 
Medicine Ltd. or any other institutions mentioned in the interview.

Financial \& competing interest's disclosure

$S$ Buono is the founder and CEO of Advanced Accelerator

Applications, and a shareholder. The interviewee has no other relevant affliations or financial involvement with any organization or entity with a financial interest in or financial conflict with the subject matter or materials discussed in the manuscript apart from those disclosed.

No writing assistance was utilized in the production of this manuscript. 